\title{
Sleep Architecture in Patients with Idiopathic Epilepsy
}

\author{
Mohamed Saad $^{1} \cdot$ Mohamed Gomaa $^{1} \cdot$ Tamer Belal $^{1} \cdot$ Wesam Fathi ${ }^{1} \cdot$ Samer Salama ${ }^{1}$ (1)
}

Received: 4 February 2019 / Accepted: 25 March 2019 / Published online: 21 April 2019

(c) The Author(s) 2019

\begin{abstract}
Purpose Studying the effects of epilepsy on sleep and its architecture and sleep on the epilepsy activity using sleep questionnaires, electroencephalogram (EEG), and polysomnogram (PSG) in patients with idiopathic epilepsy.

Methods 25 patients with idiopathic epilepsy and 25 healthy controls ( $M: F=12: 13$; age: $21.32 \pm 4.23$ years) underwent assessment with sleep questionnaires, EEG and overnight PSG. Statistical Package for the Social Sciences vs.21 was used for statistical analysis. A ' $p$ ' b 0.05 was considered as statistically significant.

Results There was poor sleep quality in patients compared to controls $(p=0.02)$, while there was no significant difference in Epworth sleepiness scale scores between the groups. There was poor sleep quality in patients with idiopathic epilepsy compared to controls.

Conclusion Patients with epilepsy activity had poor sleep quality in comparison to those with controlled disease status. Patients with juvenile myoclonic epilepsy showed initial insomnia as they had significant increase in sleep onset latency in comparison with other patients with idiopathic generalized epilepsy.
\end{abstract}

Keywords Epileptic activity $\cdot$ Parasomnia $\cdot$ Periodic limb movement $\cdot$ Refractory epilepsy $\cdot$ Antiepileptic drugs $\cdot$ Deep sleep

\begin{tabular}{ll}
\multicolumn{2}{l}{ Abbreviations } \\
AHI & Apnea-hypopnea index \\
AI & Arousal index \\
CBZ & Carbamazepine \\
ESS & Epworth sleepiness scale \\
JME & Juvenile myoclonic epilepsy \\
LEV & Levetiracetam \\
PLMI & Periodic limb movement index \\
PSG & Polysomnogram \\
PSQI & Pittsburgh sleep quality index \\
REM & Rapid eye movement \\
SWS & Slow wave sleep \\
TPM & Topiramate \\
TST & Total sleep time \\
REM & Rapid eye movement \\
VPA & Valproic acid \\
WASO & Wake after sleep onset
\end{tabular}

Samer Salama

samer_salama@mans.edu.eg

1 Department of Neurology, Mansoura University, Mansoura 25435511, Egypt

\section{Introduction}

Patients with epilepsy commonly report poor sleep quality, increased nocturnal awakenings, early morning awakenings, difficulty initiating sleep, and excessive daytime sleepiness. Nineteen percent of generalized seizures occur during sleep, as compared to $51 \%$ of localization-related seizures. One in five patients with epilepsy has seizures exclusively during sleep [1].

Most sleep-related seizures occur out of non-rapid eye movement (NREM) sleep, with NREM sleep stage N2 being the most common. This sleep stage likely facilitates focal spikes and epileptic activity through thalamocortical hyper synchrony, as represented by characteristic sleep spindles and $\mathrm{K}$ complexes [2].

Sleep deprivation has been used to provoke epilepticrelated EEG activity. Sleep itself may activate interictal activity in approximately one-third of patients with epilepsy and up to $90 \%$ of people with sleep-wake-related or statedependent epilepsies [3]. 


\section{Patients and Methods}

This is a cross-sectional study approved by the Ethical Committee of faculty of medicine, Mansoura University. The study was carried out through the period March 2016-October 2017.

The sample of this study consists of 25 patients diagnosed with idiopathic epilepsy, admitted to Neurology department in Mansoura University Hospital (MUH) through the out patients' clinic (OPC) of Neurology with age range from 15 to 40 years. Diagnosis and classification of epilepsy were based on the International League Against Epilepsy guidelines [4]. Patients with at least 1 seizure within the past 6 months of polysomnography were classified as having poor seizure control. Patients with history of underlying diseases (e.g., asthma, inborn errors of metabolism) or co-morbid illness (medical or psychiatric) or on medication affecting sleep other than antiepileptic drugs AEDs were excluded from our study.

Control group of 25 non-epileptic individuals, matching for age and sex with patients, with no psychiatric morbidity and with no complaints regarding sleep difficulties were included. The control group members were friends of the patients and hospital medical personnel on routine day duties. They were not related to any of the patients and did not have a family history of epilepsy or any other significant past medical history.

All patients and controls underwent detailed clinical, family, and treatment history, neurological examination and 16-channel electroencephalogram (EEG). Imaging of the brain was normal in all patients and controls. They were administered two validated sleep questionnaires, the Epworth Sleepiness Scale (ESS) and Pittsburgh Sleep Quality Index (PSQI). Following this evaluation, overnight polysomnogram (PSG) was recorded for only one night. During the PSG study, precautions were taken to keep the patient comfortable as far as possible.

Consents were taken from the patients (or their parents for children) indicating their acceptance to participate in the research. The consent form included the procedures to be done (EEG, MRI brain, polysomnographic study) and the statement that the confidentiality would be preserved.

\section{Results}

In our studied patients the age range was 15-32 years (mea $\mathrm{n} \pm \mathrm{SD}=21.32 \pm 4.23$ years), $55 \%$ were male and the mean BMI was $27.54 \pm 5.99$ (range 19-37.4). There were no statistically significant differences found between the studied groups ( $p$ value $>0.05$ )
Epilepsy with generalized tonic-clonic seizures alone represents $64 \%$ of our patients, coming next is juvenile myoclonic epilepsy (JME) 32\% and lastly $4 \%$ of cases suffered from childhood absence epilepsy. Sixty-four percent of patients had infrequent seizures while $36 \%$ had controlled disease status. Fourteen patients were on single AED and 11 patients were on polytherapy. Twenty-one of them were taking valproic acid (VPA), eight on levetiracetam (LEV), three on carbamazepine (CBZ) while one was taking topiramate (TPM).

Patients with idiopathic epilepsy had higher scores on PSQI than healthy controls $(p<0.001)$. ESS showed no significant differences between them $(p \geq 0.05)$. Total sleep time (TST) in patients' group was decreased in comparison with healthy controls yet it showed no statistically significant difference $(p \geq 0.05)$. Moreover, patients group had significant decrease in sleep efficiency, slow wave sleep (SWS), rapid eye movement $(\mathrm{REM}) \%(p \leq 0.001)$. Periodic limb movement index (PLMI) and wake after sleep onset (WASO) showed significant increase in patients' group than controls $(p<0.05)$ (Table 1).

Patients with uncontrolled epilepsy had statistically significant high scores in PSQI scores than controlled patients $(p \leq 0.001)$. They also showed significant decrease in (TST), N3\%, total NREM $\%$, REM $\%$ and WASO $(p \leq 0.001)$. There was no statistically significant correlation between REM latency, sleep onset latency, N1\%, N2\%, Arousal Index (AI), Apnea-Hypopnea Index (AHI) and PLMI during sleep between the two groups $(p>0.05)$ (Table 2).

Patients with JME showed high statistically significant delayed sleep onset latency in relation to patients with epilepsy with GTCS alone $(p \leq 0.001)$. There was no statistically significant correlation between TST, sleep efficiency, REM latency, N1\%, N2\%, N3\%, AI, AHI and PLMI during sleep and idiopathic epilepsy subtypes $(p>0.05)$ (Table 3$)$.

Patients who were on polytherapy had statistically significant high PSQI index than those on single AED $(p<0.05)$. While there was no statistically significant difference in ESS $(p \geq 0.05)$, they showed significant reduction in TST and sleep efficiency than those on single AED $(p<0.001)$, statistically significant reduction in REM sleep $(p<0.05)$ and statistically significant increase in WASO was detected in those on multiple AEDs $(p<0.05)$, while there was no statistically significant variation in other polysomnographic parameters (Table 4).

After logistic regression analysis of independent predictors for uncontrolled epilepsy, reduction of total sleep time, REM\% and increase in WASO were significant independent predictors (Table 5). 
Table 1 Polysomnographic findings in studied cases in comparison with control group
Table 2 Effect of epilepsy activity on sleep profile

\begin{tabular}{llll}
\hline Variables & Cases $(n=25)$ & Control $(n=25)$ & $P$ value \\
\hline Total sleep time & $5.97 \pm 1.50$ & $6.59 \pm 0.80$ & 0.077 \\
Sleep efficiency & $76.05 \pm 19.12$ & $86.00 \pm 8.96$ & $0.023^{*}$ \\
N1\% median & $6.50(2.8-13.3)$ & $7.30(2.20-19.5)$ & 0.260 \\
N2\% & $38.13 \pm 11.96$ & $42.43 \pm 4.97$ & 0.102 \\
N3\% median & $18.4(0.0-53.4)$ & $23.2(18-27.1)$ & $0.039^{*}$ \\
Rapid eye movement sleep median & $12.70(0.00-32.30)$ & $21.30(9.00-27.50)$ & $0.001^{*}$ \\
Arousal index in NREM median & $10.70(2.80-38.90)$ & $10.10(3.90-31.80)$ & 0.915 \\
Arousal index in REM median & $5.70(0.00-21.00)$ & $6.00(1.40-20.00)$ & 0.734 \\
Periodic limb movement index median & $3.60(0.00-87.60)$ & $0.70(0.00-11.30)$ & $0.010^{*}$ \\
Apnea-hypopnea index median & $0.20(0.00-7.10)$ & $0.20(0.00-1.80)$ & 0.825 \\
Sleep onset latency median & $15.36(4.03-25.46)$ & $11.60(1.81-25.46)$ & 0.065 \\
ROL mean \pm SD & $120.28 \pm 28.5$ & $111.62 \pm 34.38$ & 0.337 \\
Wake after sleep onset (in minutes) median & $32.51(5.50-78.90)$ & $16.06(2.50-60.00)$ & $0.009^{*}$ \\
\hline
\end{tabular}

\begin{tabular}{llllc}
\hline Variables & Uncontrolled $(n=9)$ & & Controlled $(n=16)$ & $P$ value \\
\cline { 2 - 2 } \cline { 5 - 6 } & No\% & & No\% & \\
\cline { 1 - 2 } Epworth sleepiness scale & $4.00 \pm 1.58$ & & $4.25 \pm 1.73$ & 0.724 \\
Pittsburgh sleep quality index & $9.00(5.00-14.00)$ & & $5.00(2.00-6.00)$ & $<0.001^{* *}$ \\
Total sleep time & $4.44 \pm 1.41$ & & $6.83 \pm 0.57$ & $<0.001^{* *}$ \\
Sleep efficiency & $55.90 \pm 16.79$ & & $87.38 \pm 7.34$ & $<0.001^{* *}$ \\
N1\% & $7.30(5.80-19.50)$ & & $7.05(2.20-15.9)$ & 0.497 \\
N2\% & $34.17 \pm 13.91$ & & $40.35 \pm 10.54$ & 0.222 \\
N3\% & $10.30(0.00-43.50)$ & & $20.75(6.60-53.40)$ & 0.101 \\
Rapid eye movement sleep & $6.40(0.00-12.70)$ & & $15.80(8.70-32.30)$ & $0.001^{*}$ \\
Arousal index in NREM & $11.20(6.70-38.90)$ & & $10.25(2.80-20.50)$ & 0.428 \\
Arousal index in REM & $5.00(0.00-11.80)$ & & $6.20(1.20-21.00)$ & 0.183 \\
Periodic limb movement index & $3.60(0.00-12.50)$ & & $3.60(0.00-87.60)$ & 0.775 \\
Apnea-hypopnea index & $0.20(0.00-1.10)$ & & $0.25(0.00-7.10)$ & 0.500 \\
Sleep onset latency & $14.73(4.48-20.48)$ & & $15.91(4.03-25.46)$ & 0.497 \\
ROL & $118.19 \pm 27.36$ & & $121.46 \pm 29.97$ & 0.790 \\
Wake after sleep onset (in minutes) & $56.70(19.40-78.90)$ & & $19.45(5.50-43.70)$ & $0.001^{*}$ \\
\hline
\end{tabular}

\section{Discussion}

Patients with idiopathic epilepsies are ideal for studying the effect of epilepsy on sleep, in view of the multiple seizure types, their strong association with sleep and vulnerability to sleep deprivation [5]. Available literature on PSG in idiopathic epilepsies has focused primarily on sleep.

It is reported that the disturbances in the sleep architecture in patients with idiopathic epilepsies may reflect the effect of epileptiform activity on normal sleep architecture $[6,7]$. This may explain the higher wake percentage, longer sleep onset latency and the reduced sleep efficiency in patients with idiopathic epilepsies noted in this study.

Epworth sleepiness scale (ESS) did not show any statistical significant difference between healthy controls and patients group ( $p \geq 0.05$ ), this goes with Ramachandraiah study [8]. In contrast to Chen and his colleagues' study where patients with uncontrolled epilepsy had significant higher ESS this may be explained as they have symptomatic epilepsy and on polytherapy and they had frequent nocturnal seizures that disturb normal nocturnal sleep leading to daytime hypersomnolence and in our study there was no patient with intractable epilepsy [9].

Patients with idiopathic epilepsy had poor sleep quality. They had higher scores on PSQI questionnaires. Also, initial insomnia increased sleep onset latency reduced sleep efficiency as they had decreased total sleep time, increased in awake after sleep onset, decreased N3 sleep\% and REM sleep\%. Similar abnormalities were noted in a cohort of medically refractory generalized epilepsy patients by Marzec and his colleagues [10]. In another study of 39 TLE 
Table 3 Relation between seizure type and other parameters in cases group

\begin{tabular}{|c|c|c|c|}
\hline \multirow[t]{2}{*}{ Variables } & \multirow{2}{*}{$\begin{array}{l}\text { Generalized epilepsy } \\
(n=16) \\
\text { No\% }\end{array}$} & \multirow{2}{*}{$\begin{array}{l}\text { Generalized myoclonic } \\
\text { epilepsy }(n=8) \\
\text { No\% }\end{array}$} & \multirow[t]{2}{*}{$P$ value } \\
\hline & & & \\
\hline Epworth sleepiness scale & $4.06 \pm 1.61$ & $3.87 \pm 1.24$ & 0.776 \\
\hline Pittsburgh sleep quality index & $5.50(2.00-14.00)$ & $5.50(3.00-12.00)$ & 0.781 \\
\hline Total sleep time & $6.23 \pm 1.18$ & $5.37 \pm 2.02$ & 0.202 \\
\hline Sleep efficiency & $80.70 \pm 15.24$ & $66.27 \pm 24.39$ & 0.088 \\
\hline $\mathrm{N} 1 \%$ & $6.65(2.20-19.50)$ & $9.20(5.80-15.90)$ & 0.209 \\
\hline $\mathrm{N} 2 \%$ & $38.47 \pm 9.76$ & $36.43 \pm 16.54$ & 0.706 \\
\hline N3\% & $21.00(0.00-53.40)$ & $9.25(6.60-43.50)$ & 0.058 \\
\hline Rapid eye movement sleep & $13.40(0.00-32.30)$ & $12.60(0.00-23.80)$ & 0.668 \\
\hline Arousal index in NREM & $9.50(2.80-38.90)$ & $12.45(7.70-20.50)$ & 0.081 \\
\hline Arousal index in REM & $5.70(0.00-20.50)$ & $5.40(0.00-21.00)$ & 0.581 \\
\hline Periodic limb movement index & $3.90(0.00-87.60)$ & $3.50(0.00-11.70)$ & 0.853 \\
\hline Apnea-hypopnea index & $0.30(0.00-3.40)$ & $0.00(0.00-7.10)$ & 0.147 \\
\hline Sleep onset latency & $13.85(4.03-24.31)$ & $18.40(11.60-24.02)$ & $0.032 *$ \\
\hline ROL & $123.31 \pm 27.92$ & $119.29 \pm 29.37$ & 0.747 \\
\hline Wake after sleep onset (in minutes) & $19.70(5.50-70.70)$ & $34.70(11.50-78.90)$ & 0.120 \\
\hline
\end{tabular}

Table 4 Effect of the numbers of AEDs on polysomnographic findings
Table 5 Logistic regression analysis of independent predictors of uncontrolled epilepsy

\begin{tabular}{llll}
\hline Variables & Single $(n=14)$ & Multiple $(n=11)$ & $P$ value \\
\hline Total sleep time & $6.76 \pm 0.84$ & $4.97 \pm 1.57$ & $0.001^{*}$ \\
Sleep efficiency & $86.24 \pm 10.8$ & $63.08 \pm 19.86$ & $0.001^{*}$ \\
N1\% median (range) & $7.05(2.2-15.9)$ & $7.3(2.9-19.5)$ & 0.661 \\
N2\% & $42.58 \pm 8.88$ & $32.46 \pm 13.33$ & $0.033^{*}$ \\
N3\% median (range) & $20(6.6-53.4)$ & $10.7(0-47.4)$ & 0.352 \\
Rapid eye movement sleep median (range) & $15.25(5.7-32.3)$ & $9(0-17.3)$ & $0.009^{*}$ \\
Arousal index in NREM median (range) & $10.35(2.8-20.5)$ & $10.7(4.4-38.9)$ & 0.827 \\
Arousal index in REM median (range) & $8.25(1.2-21)$ & $4.9(0-11.8)$ & 0.052 \\
Periodic limb movement index median (range) & $3.6(0-87.6)$ & $3.6(0-12.5)$ & 0.825 \\
Apnea-hypopnea index median (range) & $0.3(0-7.1)$ & $0.2(0-1.1)$ & 0.269 \\
Sleep onset latency median (range) & $14.34(4.03-25.46)$ & $16.08(4.48-24.02)$ & 0.622 \\
ROL mean \pm SD & $124.49 \pm 32.46$ & $114.92 \pm 22.93$ & 0.416 \\
Wake after sleep onset (in minutes) median (range) & $19.45(5.5-70.7)$ & $41(13.02-78.9)$ & $0.009^{*}$ \\
\hline
\end{tabular}

\begin{tabular}{llll}
\hline Independent predictors & \multicolumn{2}{l}{ Logistic regression analysis } \\
\cline { 2 - 4 } & $B$ & OR $(95 \% \mathrm{CI})$ & $P$ value \\
\hline Total sleep time & -3.844 & $0.021(0.001-0.72)$ & $0.033^{*}$ \\
Sleep efficiency & -0.349 & $0.71(0.49-1.01)$ & 0.056 \\
Rapid eye movement sleep & -0.411 & $0.66(0.47-0.93$ & $0.018^{*}$ \\
Wake after sleep onset (in minutes) & 0.128 & $1.13(1.03-1.25$ & $0.011^{*}$ \\
\hline
\end{tabular}

patients, all of whom showed sleep architecture fragmentation, decreased amount of REM sleep (92\%), increased number of awakenings, increased stage shifts (100\%) and increase in time awake after sleep onset (77\%). Excessive day time sleepiness (EDS) was the most frequent complaint in these patients [11].
PLMI showed statistically significant increase in patients' group than in controls $(p<0.05)$. This is in contrast to the findings in Ramachandraiah and Krishnan's studies which studied polysomnographic findings in JME patients this could be explained as our patients were not exclusively with JME but also other forms of idiopathic 
epilepsies were included also not all the patients were on the same AED or on monotherapy [8, 12]. In Maganti and his colleagues study there was also no statistically significant increase in PLMI between patients and control that could be explained by small number of studied patients [11] and their relative younger mean age 13.36 years [13].

Patients with epilepsy activity have poor sleep quality that was evident by having higher PSQI scores, decreased SWS, REM\% and increase in nocturnal awaking from sleep. This goes with the findings of Chen and his colleagues where patients with poor seizure had high PSQI scores more than 5 in comparison with those with controlled epilepsy ( $p$ value 0.019) [9].

Sleep respiratory parameters do not show significant differences between patients and healthy subjects and between patients with controlled epilepsy and others who have infrequent seizures. This can be explained by the followings: our patients had BMI within normal and no hypertrophied tonsils. This goes with the finding in Ramachandraiah and also Maganti's studies who studied Sleep Architecture in 11 Children with Idiopathic Generalized Epilepsy and six healthy controls [8]. In contrast with the findings in Gogou and his colleagues study which studied 40 children with idiopathic epilepsy [Mean age (10.61 \pm 2.41$)$ ] and 27 healthy children [mean age $(11 \pm 2)]$ where there were statistically significant increases in OAI and OAHI in patients group [14]. This can be explained by the fact that they had higher BMI than our study and they had hypertrophic adenoid or tonsils.

Patients with JME have initial insomnia. They showed statistically significant delayed sleep onset latency compared with other patients with idiopathic epilepsy. This goes with Krishnan and his colleague's study: a study in which 25 patients with JME and 25 healthy controls were evaluated and it demonstrated delayed sleep onset ( $p$ value 0.039) [12]. The reason for longer sleep latency in JME could be due to evening circadian chronotype [15].

In our study not all the patients took the same drug and some of them took single drugs, others were on polytherapy. This study is probably the only one to analyze the effect of polytherapy on sleep in idiopathic epilepsy patients. Patients who were on polytherapy had poor sleep quality, they had higher PSQI index scores. Also, significant reduction in total sleep time and sleep efficiency, REM sleep and statistically significant increase in WASO resulting in maintenance insomnia. However, in another study, treatment of newly diagnosed epilepsy patients with carbamazepine for a month resulted in improvement of the abnormal sleep parameters [16]. This may reflect the importance of early treatment and proper control of epilepsy activity.

\section{Summary}

Patients with idiopathic epilepsy are frequently complaining about poor sleep quality. Epilepsy syndrome, number of AEDs, and epilepsy activity were the commonest predisposing factors to poor sleep quality. Measures should be taken to improve quality of sleep in these patients including proper diagnoses of epilepsy syndrome, choice of AED, use monotherapy as much as possible and control epilepsy activity.

Funding This study was supported by the Department of Neurology, Faculty of Medicine at Mansoura University.

\section{Compliance with Ethical Standards}

Conflict of Interest All authors declare that they have no conflict of interest.

Ethical Approval All procedures performed in studies involving human participants were in accordance with the ethical standards of the institutional research committee and with the 1964 Helsinki Declaration and its later amendments or comparable ethical standards.

Informed Consent Informed consent was obtained from all individual participants included in the study.

Open Access This article is distributed under the terms of the Creative Commons Attribution 4.0 International License (http://creativeco mmons.org/licenses/by/4.0/), which permits unrestricted use, distribution, and reproduction in any medium, provided you give appropriate credit to the original author(s) and the source, provide a link to the Creative Commons license, and indicate if changes were made.

\section{References}

1. Sinha S, Brady M, Scott C, Walker M. Do seizures in patients with refractory epilepsy vary between wakefulness and sleep? J Neurol Neurosurg Psychiatry. 2006;77(9):1076-8.

2. Buechler RD, Rodriguez AJ, Lahr BD, So EL. Ictal scalp EEG recording during sleep and wakefulness: diagnostic implications for seizure localization and lateralization. Epilepsia. 2008;49(2):340-2.

3. Eriksson SH. Epilepsy and sleep. Curr Opin Neurol. 2011;24(2):171-6.

4. Iliescu C, Craiu D. Diagnostic approach of epilepsy in childhood and adolescence. Maedica. 2013;8(2):195.

5. Panayiotopoulos C. Epileptic syndromes and their treatment. Neonatal seizures. Second edition. London; 2007. p. 185-206.

6. Bonakis A, Koutroumanidis M. Epileptic discharges and phasic sleep phenomena in patients with juvenile myoclonic epilepsy. Epilepsia. 2009;50(11):2434-45.

7. Gigli G, Calia E, Marciani M, Mazza S, Mennuni G, Diomedi $\mathrm{M}$, et al. Sleep microstructure and EEG epileptiform activity in patients with juvenile myoclonic epilepsy. Epilepsia. 1992;33(5):799-804. 
8. Ramachandraiah C, Sinha S, Taly A, Rao S, Satishchandra $\mathrm{P}$. Interrelationship of sleep and juvenile myoclonic epilepsy (JME): a sleep questionnaire-, EEG-, and polysomnography (PSG)-based prospective case-control study. Epilepsy Behav. 2012;25(3):391-6.

9. Chen N-C, Tsai M-H, Chang C-C, Lu C-H, Chang W-N, Lai $\mathrm{S}-\mathrm{L}$, et al. Sleep quality and daytime sleepiness in patients with epilepsy. Acta Neurol Taiwan. 2011;20(4):249-56.

10. Marzec ML, Selwa LM, Malow BA. Analysis of the first night effect and sleep parameters in medically refractory epilepsy patients. Sleep Med. 2005;6(3):277-80.

11. de Almeida C, Lins OG, Lins SG, Laurentino S, Valença MM. Distúrbios do sono na epilepsia do lobo temporal. Arq Neuropsiquiatr. 2003;61(4):979-87.

12. Krishnan P, Sinha S, Taly AB, Ramachandraiah CT, Rao S, Satishchandra P. Altered polysomnographic profile in juvenile myoclonic epilepsy. Epilepsy Res. 2014;108(3):459-67.
13. Maganti R, Sheth RD, Hermann BP, Weber S, Gidal BE, Fine J. Sleep architecture in children with idiopathic generalized epilepsy. Epilepsia. 2005;46(1):104-9.

14. Gogou M, Haidopoulou K, Eboriadou M, Pavlidou E, Hatzistylianou M, Pavlou E. Sleep respiratory parameters in children with idiopathic epilepsy: a cross-sectional study. Epilepsy Res. 2016;126:10-5.

15. Pung T, Schmitz B. Circadian rhythm and personality profile in juvenile myoclonic epilepsy. Epilepsia. 2006;47(s2):111-4.

16. Touchon J, Billiard M, Baldymoulinier M, Besset A, Cadilhac J. Sleep and Partial Epilepsy. Sleep 1989;88:147-50.

Publisher's Note Springer Nature remains neutral with regard to jurisdictional claims in published maps and institutional affiliations. 